\section{Consumo alimentar de crianças menores de três anos residentes em área de alta prevalência de insegurança alimentar domiciliar}

\author{
Food intake among children under three years of \\ age in an area with high food insecurity
}

\author{
1 Instituto de Nutrição Josué \\ de Castro, Universidade \\ Federal do Rio de Janeiro, \\ Rio de Janeiro, Brasil. \\ 2 Instituto de Medicina \\ Social, Universidade do \\ Estado do Rio de Janeiro, \\ Rio de Janeiro, Brasil. \\ Correspondência \\ R. Salles-Costa \\ Instituto de Nutrição Josué de \\ Castro, Universidade Federal \\ do Rio de Janeiro. \\ Cidade Universitária, \\ Av. Carlos Chagas Filho \\ 373, Edifício do Centro de \\ Ciências da Saúde, Bloco J, \\ 2 o andar, Rio de Janeiro, RJ \\ 21941-902, Brasil. \\ rosana@nutricao.ufrj.br
}

\begin{abstract}
This study focused on the association between food insecurity and children's dietary intake in a representative sample of children in an area with high food insecurity in Duque de Caxias, Rio de Janeiro State, Brazil. This was a cross-sectional, population-based study with a probabilistic cluster sample of 402 families with children ranging from six to 30 months of age. Food insecurity was assessed based on the Brazilian Food Insecurity Scale (EBIA), and children's dietary intake was evaluated with two 24-hour recalls. Intake levels for food groups, energy, and nutrients were compared according to food insecurity status. Intake of sugar/sweets and fat was high, regardless of food insecurity status, and coffee intake was significantly higher among children with food insecurity. In the energy-adjusted linear regression model, protein intake was inversely associated with the EBIA ( $p=0.005)$. The results suggest that family food insecurity jeopardizes the quality of children's diet, reducing the consumption of protein and increasing the consumption of coffee and high energy density foods.
\end{abstract}

Food Security; Food Consumption; Child
Marina Maria Leite Antunes 1

Rosely Sichieri 2

Rosana Salles-Costa 1

\section{Introdução}

A Segurança Alimentar e Nutricional é considerada como um direito humano, um bem público que se realiza por intermédio de políticas universais, garantido na Lei Orgânica de Segurança Alimentar e Nutricional no Brasil, aprovada em 2006 1. Burlandy 2 destacou que o conceito ampliado de Segurança Alimentar e Nutricional articula a dimensão alimentar, que engloba a produção, a comercialização e o consumo de alimentos, e a dimensão nutricional, referente à utilização do alimento pelo organismo e sua relação com a saúde.

Situações de insegurança alimentar podem ser detectadas por diferentes tipos de problemas tais como fome, obesidade, doenças associadas à má alimentação, consumo de alimentos de qualidade duvidosa ou prejudicial à saúde, entre outras 3. Com isso, identificar os determinantes da insegurança alimentar contribui para a melhor compreensão sobre quais grupos populacionais se apresentam mais suscetíveis ao problema além das possíveis ações para a sua redução 4 , permitindo o planejamento de ações direcionadas para as dimensões da disponibilidade de alimentos, do acesso e do consumo 5 .

Estudos internacionais descrevem a maior suscetibilidade de grupos populacionais à insegurança alimentar, sendo eles: indivíduos de origem latina e de raça/cor da pele preta e parda 6,7 , população feminina ${ }^{8}$ e, sobretudo, famílias com 
crianças na sua composição ${ }^{9}$. O estudo realizado por Nnakwe \& Yegammia 10, em Coimbatore, na Índia, revelou prevalência significativamente maior de insegurança alimentar entre famílias com crianças nos domicílios (57\%), quando comparadas com aquelas sem crianças (44\%). Também nos Estados Unidos, a prevalência de insegurança alimentar se revela maior entre as famílias com crianças menores de seis anos $(16,7 \%)^{9}$.

No Brasil, a Escala Brasileira de Insegurança Alimentar (EBIA) é o instrumento utilizado para estimar a situação de insegurança alimentar na população ${ }^{11}$. Trata-se de um questionário validado com base na metodologia desenvolvida e utilizada pela Universidade de Cornell (Ithaca, Estados Unidos) para avaliação da insegurança alimentar familiar na população norte-americana. Entre os estudos de base populacional que utilizaram a EBIA realizados no Brasil, dois trabalhos merecem destaque. O primeiro deles foi a Pesquisa Nacional por Amostra de Domicílios (PNAD), feita em 2004 12, que revelou cerca de $35 \%$ de famílias em situação de insegurança alimentar. O segundo estudo, a Pesquisa Nacional sobre Demografia e Saúde (PNDS) 13, executada em 2006, avaliou o perfil da população feminina em idade fértil e de crianças até cinco anos de idade da população brasileira. Neste estudo, a prevalência de insegurança alimentar, estimada pela EBIA, foi próxima a 38\%, permaneceu em proporções mais elevadas entre famílias com crianças ( $57 \%$ vs. $44 \%)$.

Considerando o conceito ampliado de Segurança Alimentar e Nutricional, Burlandy \& SallesCosta 14 destacaram recentemente que a redução na disponibilidade de alimentos, tende a comprometer a qualidade e a quantidade dos alimentos que compõem a cesta básica das famílias em situação de insegurança alimentar. Tal quadro, quando associado ao desenvolvimento infantil, revela um comprometimento do aporte de energia e nutrientes, intensificando a deficiência do aporte de nutrientes 4,6,15, refletindo no déficit de crescimento de crianças 16.

Dessa forma, considerando que a influência da insegurança alimentar no consumo de alimentos representa um importante pilar na investigação da situação nutricional das crianças, o objetivo deste trabalho foi descrever a associação entre insegurança e consumo alimentar de crianças.

\section{Material e métodos}

\section{População de estudo}

Trata-se de um inquérito com amostragem probabilística, em conglomerados, de domicílios particulares permanentes do Distrito de Campos Elíseos, Município de Duque de Caxias, Rio de Janeiro, Brasil. Foi utilizada uma amostra probabilística de 1.125 domicílios, selecionada em três estágios (setor censitário, domicílio e o indivíduo). O tamanho da amostra foi fixado com base em uma estimativa de $14,5 \%$ para a prevalência de pobreza extrema, fixando-se um erro relativo máximo de $5 \%$. No primeiro estágio, foram selecionados, de forma sistemática, 75 setores censitários com probabilidades proporcionais ao número de domicílios particulares permanentes observados pelo Censo Demográfico 2000, dentre os 322 setores constantes da Base Operacional Geográfica (BOG) de 2000 do Instituto Brasileiro de Geografia e Estatística (IBGE). Previamente à seleção, os setores censitários foram ordenados por renda média do responsável pelo domicílio, de forma a assegurar a representação de todos os níveis de renda na amostra. Em cada setor foram selecionados 15 domicílios e em cada domicílio foi entrevistada uma pessoa de cada faixa etária (adulto, adolescente e criança), também selecionada aleatoriamente.

O tamanho final da amostra foi de 1.085 domicílios, com $4 \%$ de perdas $(n=45)$, e $37 \%$ desses domicílios ( $\mathrm{n}=402$ ) apresentavam crianças na faixa etária de estudo do presente trabalho. Considerando a estimativa inicial de 435 domicílios com crianças de 6 a 30 meses, a amostra final de crianças apresentou uma perda de 7,6\%. Maiores detalhes do desenho da amostra e controle de qualidade do estudo encontram-se publicados em Salles-Costa et al. ${ }^{17}$.

A população estudada foi composta por $72 \%$ das famílias em situação de insegurança alimentar: $39 \%$ apresentavam insegurança alimentar leve, $21 \%$ insegurança alimentar moderada e $12 \%$ insegurança alimentar grave, com proporção de $3 \%$ dos chefes das famílias sem escolaridade, enquanto cerca de $75 \%$ não concluíram o ensino fundamental e proporção inferior a $1 \%$ concluiu o ensino universitário. E $23 \%$ das famílias referiram renda per capita acima de um salário-mínimo ( $\mathrm{R} \$ 300,00$ na época do estudo) 16.

A coleta de dados foi realizada no período de maio a dezembro de 2005 e as informações sobre o domicílio foram respondidas pelo chefe da família ou pelo responsável que permanecia mais tempo em casa e cuidava da alimentação. 


\section{Avaliação da insegurança alimentar}

Utilizou-se a EBIA 11, que consta de 15 perguntas centrais fechadas que admitem sim ou não como resposta, e que abordam desde a preocupação da comida acabar antes de se poder comprar mais até a ausência total dela, nos três meses precedentes à entrevista. Das 15 perguntas, sete se referem a membros da família menores de 18 anos. Cada resposta afirmativa do questionário de insegurança alimentar corresponde a "um ponto"; e para as famílias com a faixa etária em questão, a classificação é dada nos seguintes níveis: (a) segurança alimentar - nenhum ponto; (b) insegurança alimentar leve - 1 a 5 pontos; (c) insegurança alimentar moderada - 6 a 10; e (d) insegurança alimentar grave -11 a 15 pontos 18 . Cabe ressaltar que a EBIA avalia Segurança Alimentar e Nutricional apenas pela dimensão do acesso à aquisição de alimentos; logo, o termo Segurança Alimentar se apresenta mais adequado, pois exclui a dimensão nutricional não avaliada pelo referido método.

\section{Consumo alimentar}

Os dados foram coletados por seis nutricionistas treinados, por meio de dois recordatórios de 24 horas, realizados em dias alternados no período máximo de um mês entre eles, estimando o consumo. As porções relatadas, valendo-se de medidas caseiras (copos, pratos, talheres, mamadeiras), foram convertidas em gramas e mililitros, $\mathrm{e}$ o consumo de energia e nutrientes foi estimado com o auxílio do Programa de Apoio à Nutrição NUTWIN, do Departamento de Informática em Saúde da Universidade Federal de São Paulo conforme descrito em Salles-Costa et al. 19.

As análises foram desenvolvidas com base nas médias dos dois recordatórios para energia (kcal), de macronutrientes (gramas e percentual em relação à energia total), de micronutrientes (miligramas) e de grupos de alimentos (porções).

Os alimentos relatados foram classificados em oito grupos propostos pelo Ministério da Saúde 20, sendo eles: cereais, verduras e legumes, frutas, leite e derivados, carnes e ovos, leguminosas, óleos e gorduras, açúcares e doces. Para determinar a gramatura e o número das porções recomendadas foram adotados os valores propostos por Philippi et al. 21. Quanto ao consumo de café, o "café com leite pingado" foi definido como uma porção de café com 20 mililitros, considerando a preparação com $80 \%$ de leite e $20 \%$ de café.

A adequação da ingestão de energia e de micronutrientes foi baseada nas necessidades energéticas, de acordo com o sexo e a faixa etá- ria, recomendadas pelo Instituto de Medicina dos Estados Unidos 22. Consideram-se os pontos de corte de $90 \%$ e $110 \%$ para estimar o consumo abaixo e acima do recomendado de energia, respectivamente. Para avaliar a adequação da distribuição percentual dos macronutrientes em relação à energia total consumida, foram adotados os pontos de corte propostos pelo Ministério da Saúde de 200220 para crianças entre 6 e 24 meses e do Ministério da Saúde de 200623 para aquelas com idade entre 25 e 30 meses.

\section{Análise de dados}

As prevalências de adequação e inadequação do consumo de energia e nutrientes, bem como do consumo de porções dos grupos de alimentos, foram comparadas de acordo com a situação de insegurança alimentar da família com base na EBIA em dois grupos: segurança alimentar versus famílias com insegurança alimentar leve e insegurança alimentar moderada/grave. Optou-se por considerar as famílias com as formas mais graves de insegurança alimentar na mesma categoria por apresentarem características semelhantes. Em seguida, as médias das porções dos alimentos foram comparadas de acordo com o grau de insegurança alimentar. Para testar a normalidade da distribuição das variáveis do consumo alimentar investigada, utilizou-se o teste de Shapiro-Wilk W. Utilizou-se o teste t de Sudent para comparação das médias, e o teste qui-quadrado para as prevalências e proporções.

Para avaliar a influência da insegurança alimentar, no consumo dietético das crianças, usou-se regressão linear, tendo como variável independente a insegurança alimentar com o valor da pontuação final de cada domicílio pela EBIA que variou de 0 a 15 pontos, sem categorização. Esta opção foi adotada para possibilitar a observação da associação do consumo alimentar com o agravamento da situação de insegurança alimentar (aumento na pontuação de respostas positivas), independentemente da classificação em insegurança alimentar leve, insegurança alimentar moderada e insegurança alimentar grave no domicílio.

Como variáveis dependentes, foram considerados os valores de consumo de energia ( $\mathrm{kcal}$ ), proteínas (gramas) e de ferro (miligramas); a escolha desses elementos da dieta decorreu de sua relação com as deficiências nutricionais de maior relevância epidemiológica na faixa etária de estudo. $\mathrm{O}$ modelo final incluiu o ajuste por energia para o consumo de proteínas e ferro.

A análise dos dados considerou a expansão dos dados e o efeito do desenho amostral, ten- 
do como nível de significância o nível de 95\% $(\mathrm{p}<0,05)$. Todas as análises foram realizadas no pacote estatístico Stata 9.0 (Stata Corp., College Station, Estados Unidos).

Este estudo foi aprovado pelo Comitê de Ética em Pesquisa da Universidade Estadual do Rio de Janeiro em 24 de agosto de 2004.

\section{Resultados}

Das 402 crianças avaliadas, foram excluídas dez por apresentarem apenas um recordatório de 24 horas $(2,3 \%)$, cinco crianças $(1,1 \%)$ que relataram consumo de energia considerado extremamente baixo em razão do predomínio do aleitamento materno e três crianças $(0,7 \%)$ cujo consumo energético foi acima do percentil 97 da distribuição, o que resultou em $11,7 \%$ de perdas $(n=18)$. Dessa forma, a amostra final foi composta por 384 crianças, sendo 194 (50,5\%) do sexo masculino e 190 (49,5\%) do sexo feminino.

Comparando-se o percentual de inadequação do consumo de energia e nutrientes de acordo com a presença de insegurança alimentar, observou-se que a proporção de crianças em situação de segurança alimentar e que não atingiram as recomendações de consumo de proteínas apresentou-se significativamente menor quando comparadas àquela em insegurança alimentar moderada e grave. $\mathrm{O}$ mesmo quadro foi encontrado para o consumo de ferro, dado que as crianças com algum grau de insegurança alimentar apresentaram proporções maiores de inadequação do consumo desse nutriente quando comparadas àquelas em situação de segurança alimentar. A proporção de crianças em situação de insegurança alimentar moderada e insegurança alimentar grave e com consumo de glicídios acima da recomendação foi significativamente maior quando comparadas àquelas em situação de segurança alimentar (Tabela 1).

Observando-se o consumo médio de porções dos grupos de alimentos segundo o grau de insegurança alimentar (Figura 1), crianças com insegurança alimentar leve apresentaram consumo significativamente maior das porções dos grupos das hortaliças, doces e açúcares e café e consumo menor para os cereais, quando comparados às crianças de famílias em situação de segurança alimentar. Entre as crianças de famílias com as formas moderada e grave de insegurança alimentar, o consumo médio de porções apresentou-se significativamente reduzido para cereais, hortaliças, frutas, carnes e ovos e gorduras. $\mathrm{O}$ consumo de café foi mais elevado entre crianças com as formas mais severas de insegurança alimentar $(\mathrm{p}<0,05)$. Destaca-se que o consumo dos alimentos dos grupos das verduras e legumes e leite e derivados demonstrou-se aquém das recomendações, independentemente da presença da insegurança alimentar nos domicílios.

O modelo de regressão linear permitiu constatar que o consumo de energia de proteínas e de ferro apresentou-se associado significativa e negativamente com o grau de insegurança alimentar da família, de modo que quanto maior a pontuação da família na EBIA menor o consumo de energia e nutrientes pela criança. Após o ajuste por energia, o consumo de proteínas foi associado de forma inversa e significativa com insegurança alimentar (Tabela 2).

\section{Discussão}

No Brasil, a insegurança alimentar e o consumo alimentar foram avaliados em estudos como o de Fávaro et al. 24 realizado com população indígena residentes em Mato Grosso do Sul que revelou que as crianças apresentaram consumo inadequado em relação à energia em cerca de $67 \%$ das crianças pertencentes às famílias com segurança alimentar/insegurança alimentar leve e em $94,4 \%$ das crianças de famílias com insegurança alimentar moderada e/ou insegurança alimentar grave. No mesmo estudo a proporção de crianças com consumo protéico inadequado foi significativamente menor entre as crianças das famílias classificadas em segurança alimentar/insegurança alimentar leve quando comparadas às famílias com as formas mais graves de insegurança alimentar. No estudo realizado recentemente entre famílias brasileiras beneficiadas pelo Programa Bolsa Família (PBF), Segall-Corrêa \& Salles-Costa 25 ressaltaram que as famílias revelaram a percepção de aumento na quantidade e na variedade dos alimentos por causa do PBF, independentemente do grau de insegurança alimentar, e que a maior inclusão de alimentos de maior densidade calórica e menor valor nutritivo prevaleceram na decisão de consumo. No estudo realizado em Campinas por Panigassi et al. 26, os autores verificaram que a proporção de famílias em situação de insegurança, cujo informante não consumia leite e derivados, frutas, verduras/legumes, e carnes foi significantemente maior do que aquelas em situação de segurança alimentar.

No presente trabalho, a proporção de crianças com inadequação do ferro na dieta, especialmente entre aquelas com as formas mais graves de insegurança alimentar, merece ser destacada, uma vez que a anemia permanece como uma das principais morbidades endêmicas no Brasil, particularmente entre crianças, visto que políticas 
Tabela 1

Percentuais (\%) de inadequação do consumo de energia e nutrientes de crianças de 6 a 30 meses de acordo com presença de insegurança alimentar * na família. Duque de Caxias, Rio de Janeiro, Brasil, 2005.

\begin{tabular}{|c|c|c|c|c|c|c|}
\hline \multirow[t]{2}{*}{ Variáveis } & \multicolumn{3}{|c|}{ Abaixo da recomendação (\%) } & \multicolumn{3}{|c|}{ Acima da recomendação (\%) } \\
\hline & $\begin{array}{l}\text { Segurança } \\
\text { alimentar } \\
(n=111)\end{array}$ & $\begin{array}{c}\text { Insegurança } \\
\text { alimentar } \\
\text { leve } \\
(n=133)\end{array}$ & $\begin{array}{c}\text { Insegurança } \\
\text { alimentar } \\
\text { moderada e } \\
\text { grave } \\
(n=140)\end{array}$ & $\begin{array}{l}\text { Segurança } \\
\text { alimentar } \\
(\mathrm{n}=111)\end{array}$ & $\begin{array}{c}\text { Insegurança } \\
\text { alimentar } \\
\text { leve } \\
(n=133)\end{array}$ & $\begin{array}{c}\text { Insegurança } \\
\text { alimentar } \\
\text { moderada e } \\
\text { grave } \\
(n=140)\end{array}$ \\
\hline Energia ** (kcal) & 28,3 & 26,7 & 30,5 & 58,1 & 50,1 & $44,4 \# \#$ \\
\hline Proteínas *** (g) & 3,6 & $8,9 \#$ & $16,6 \#$ & 47,7 & 51,5 & 27,5 \#\# \\
\hline 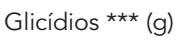 & 24,4 & 22,9 & 17,7 & 0,8 & $7,9 \#$ & $7,6 \# \#$ \\
\hline 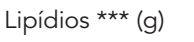 & 1,1 & 7,0 & 5,6 & 13,4 & 16,2 & 14,0 \\
\hline Ferro ** $(\mathrm{mg})$ & 33,6 & 45,9 & 64,3 \#\# & - & - & - \\
\hline
\end{tabular}

* Avaliado pela Escala Brasileira de Insegurança Alimentar 11 nas categorias de segurança alimentar, insegurança alimentar leve e insegurança alimentar moderada e grave;

** Recomendações do Instituto de Medicina dos Estados Unidos 22 de acordo com sexo e idade;

*** Recomendações do Ministério da Saúde 23 para crianças;

\# Teste qui-quadrado entre as categorias de segurança alimentar versus insegurança alimentar leve, sendo $p<0,05$;

\#\# Teste qui-quadrado entre as categorias de segurança alimentar versus insegurança alimentar moderada e grave, sendo $\mathrm{p}<0,05$.

Figura 1

Consumo alimentar em porções de acordo com o grau de insegurança alimentar $(n=384)$. Duque de Caxias, Rio de Janeiro, Brasil, 2005.

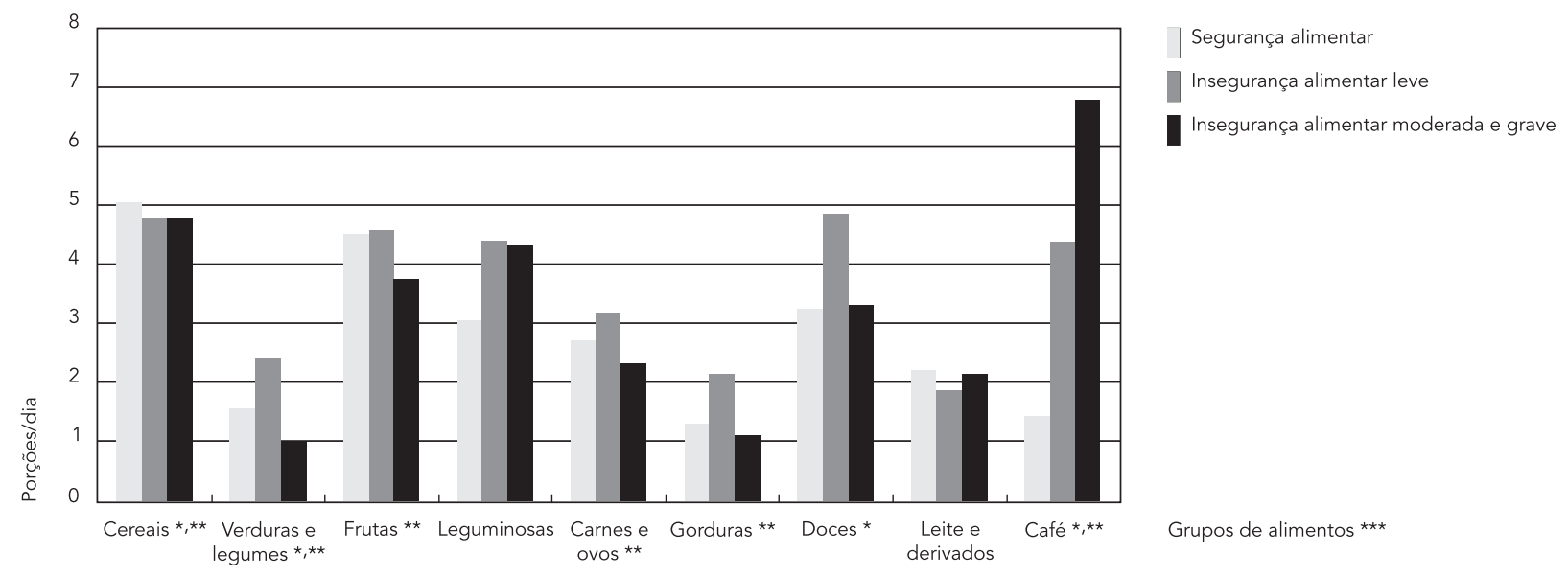

* Teste $t$ de Student entre as categorias de segurança alimentar versus insegurança alimentar leve, sendo valor de $p<0,05$;

** Teste $t$ de Student entre as categorias de segurança alimentar versus insegurança alimentar moderada + grave, sendo valor de $p<0,05$;

*** Recomendação de porções diárias de acordo com o Ministério da Saúde: cereais (3 a 5), verduras e legumes (3), frutas (3 a 4), leguminosas (1), carnes e ovos (2), óleos e gorduras (2), açúcares e doces (1) e leite e derivados (3). 
Percentuais (\%) de inadequação do consumo de energia e nutrientes de crianças de 6 a 30 meses de acordo com presença de insegurança alimentar * na família. Duque de Caxias, Rio de Janeiro, Brasil, 2005.

\begin{tabular}{|c|c|c|c|c|c|c|}
\hline \multirow[t]{2}{*}{ Variáveis dependentes } & \multicolumn{3}{|c|}{ Univariado } & \multicolumn{3}{|c|}{ Ajustado pelo consumo energético } \\
\hline & $\beta$ & IC95\% & Valor de p & $\beta$ & IC95\% & Valor de $p$ \\
\hline Energia (kcal) & $-86,4$ & $-133,8 ;-39,0$ & 0,001 & - & - & - \\
\hline Proteínas (g) & $-4,9$ & $-6,6 ;-3,1$ & $<0,001$ & $-1,9$ & $-3,3 ;-0,6$ & 0,005 \\
\hline Ferro (mg) & $-0,9$ & $-1,3 ;-0,4$ & $<0,001$ & $-0,3$ & $-0,7 ; 0,1$ & 0,09 \\
\hline
\end{tabular}

* Escala Brasileira de Insegurança Alimentar 11, avaliada como variável contínua, variando de 0 a 15 pontos.

públicas em vigor ainda não conseguem controlar essa morbidade 27. De modo semelhante, no estudo realizado entre crianças norte-americanas por Skalicky et al. ${ }^{28}$, os autores observaram que a presença de insegurança alimentar na família aumentava a chance de a criança apresentar anemia por deficiência de ferro $(\mathrm{OR}=2,4$; IC95\%: 1,1-5,2; $\mathrm{p}=0,02$ ).

Os dados de consumo alimentar encontrados corroboram com o estudo de Borges et al. 29, realizado entre crianças atendidas em uma unidade ambulatorial localizada no mesmo distrito do presente estudo, que revelou prevalência de $13 \%$ de anemia avaliada por meio de dosagem de ferro sérico. O consumo elevado de café nessas crianças pode ser um dos fatores a contribuir para o aumento da prevalência de anemia, pois os polifenóis encontrados em altas concentrações no café reduzem a absorção do ferro ${ }^{30}$. Apesar da ausência de recomendação nos guias nacionais quanto ao consumo desse alimento, é comum a presença da combinação "café com leite" entre crianças brasileiras, em particular no desjejum e no lanche da tarde, sugerindo a necessidade de recomendações de ingestão desse alimento.

A análise por meio dos grupos de alimentos revelou que as crianças não atingiram as recomendações do consumo das porções de alimentos com proteínas de alto valor biológico (leites e derivados), e hortaliças, de acordo com as recomendações do guia nacional 20 e apresentaram consumo elevado dos alimentos de alta densidade calórica (gorduras e açúcares). No Brasil, observou-se a transformação nos hábitos alimentares da população com o aumento do consumo de óleos vegetais e margarina, açúcar e em geral alimentos com alta densidade energética como biscoitos e refrigerantes, como observado na Pesquisa de Orçamentos Familiares (POF) ${ }^{31} \mathrm{de}$ 2003. Adicionalmente, Tuma et al. 32 observaram, em estudo realizado em creches de Brasília, a introdução precoce de alimentos como fast-foods, refrigerantes, enlatados e doces ou guloseimas. Padrões semelhantes de consumo foram encontrados em diversos estudos nacionais 33,34,35.

Insegurança alimentar nas suas formas mais graves (moderada e grave) contribuiu para a redução de todos os grupos de alimentos, aumentando ainda mais a inadequação dietética observada em crianças. Feinberg et al. ${ }^{36}$, avaliando famílias com crianças de 2 a 13 anos de idade, constataram associação entre a situação de insegurança alimentar e prática alimentares compensatórias, como a restrição de alimentos e adição de açúcar em bebidas.

Os padrões inadequados de consumo têm sido considerados como um dos principais fatores responsáveis pelo aumento da prevalência de obesidade infantil 37 . Um reflexo desse padrão revelou-se recentemente na PNDS 13, já que cerca de $7 \%$ das crianças com idade inferior a cinco anos já apresentavam excesso de peso, tendo em conta o índice antropométrico peso-para-altura. Em um estudo realizado em Pelotas, observouse que entre famílias com insegurança alimentar coexistem situações de excesso de peso/obesidade e déficit de peso, fato que indica tanto a diminuição da quantidade de alimentos como a perda da qualidade nutritiva 38 .

A escolha alimentar de um indivíduo engloba diversos aspectos que vão além do poder de compra da família, levando em consideração as representações do alimento para a família, o que configura um desafio na avaliação do consumo alimentar, bem como para a educação alimentar. Em estudo, Segall-Corrêa \& Salles-Costa 25 encontraram um percentual importante de famílias que após serem incluídas no programa de transferência de renda de nosso país, o PBF, referiram aumento significativo na quantidade e variedade de alimentos consumidos. Entretanto, as autoras frisaram que o aumento do poder de compra das famílias não refletiu um benefício na qualidade da dieta ingerida porque houve aumento no con- 
sumo de alimentos de alta densidade energética. As autoras reforçaram a importância de medidas de educação nutricional associadas à melhoria no acesso aos alimentos.

Cabe salientar que não existe método ouro para a avaliação do consumo alimentar, especialmente na população infantil. O recordatório de 24 horas vem sendo o método mais utilizado na faixa etária estudada aqui e se apresentou adequado em um estudo realizado em uma subamostra da população do presente artigo 39 . Ressalta-se, ainda, que a elevada prevalência de insegurança alimentar deve ser considerada ao avaliar as associações encontradas no presente estudo, sendo comparáveis apenas entre populações com características semelhantes.

\section{Resumo}

O objetivo deste artigo foi descrever a associação entre insegurança alimentar e consumo alimentar de crianças no Município de Duque de Caxias, Rio de Janeiro, Brasil. Trata-se de estudo transversal de base populacional, com amostra probabilística de domicílios; com 402 famílias compostas por crianças de 6 e 30 meses de idade. Insegurança alimentar foi avaliada com base $n a$ Escala Brasileira de Insegurança Alimentar (EBIA) e o consumo alimentar das crianças foi estimado por dois recordatórios de 24 horas. Comparou-se o consumo dos grupos de alimentos, de energia e de nutrientes entre as categorias de insegurança alimentar. O consumo de doces e de gorduras foi elevado, independentemente do grau de insegurança alimentar, e o consumo de café foi significativamente maior entre crianças com insegurança alimentar. No modelo de regressão linear ajustado por energia, o consumo de proteínas associou-se de forma inversa com a EBIA ( $p=0,005)$. Os resultados encontrados sugerem que a insegurança alimentar das famílias compromete a qualidade da dieta infantil, reduzindo o consumo de alimentos protéicos, aumentando o consumo de café e de alimentos de alta densidade energética.

Segurança Alimentar e Nutricional; Consumo de Alimentos; Criança
Em conclusão, as crianças avaliadas neste estudo apresentaram elevada ingestão de alimentos de alta densidade calórica e consumo aquém das recomendações de leites e derivados, e de hortaliças ratificando a tendência constatada nos últimos anos no Brasil. Esse perfil de consumo alimentar contribuiu para a ingestão inadequada de energia e nutrientes, sobretudo de ferro. Em conjunto, os achados reforçam a importância de ações de combate à insegurança alimentar, bem como ações educativas para a melhoria da qualidade da dieta ingerida pela população como um todo e, em particular, para as famílias em insegurança alimentar.

\section{Colaboradores}

M. M. L. Antunes participou na coleta, análise dos dados e redação do artigo. R. Sichieri idealizou a proposta da pesquisa, foi responsável pelo financiamento, participou de todas as etapas de elaboração do estudo, análise de dados e revisão da versão final do artigo. R. Salles-Costa foi responsável pelo desenho do estudo, coordenação da investigação de campo, desenho, análise e interpretação de dados, redação e aprovação da versão final do artigo.

\section{Agradecimentos}

Ao Conselho Nacional de Desenvolvimento Científico e Tecnológico (CNPq, processo no. 503139/2003-3) e Instituto Nacional de Câncer pelo apoio financeiro. 


\section{Referências}

1. Brasil. Lei $\mathrm{n}^{\circ}$. 11.346, de 15 de setembro de 2006 Cria o Sistema Nacional de Segurança Alimentar e Nutricional - SISAN com vistas em assegurar o direito humano à alimentação adequada e dá outras providências. Diário Oficial da União 2006; 18 set.

2. Burlandy L. Transferência condicionada de renda e segurança alimentar e nutricional. Ciênc Saúde Coletiva 2007; 12:1441-51.

3. Conselho Nacional de Segurança Alimentar. Documento base da III Conferência Nacional de Segurança Alimentar e Nutricional. Brasília: Conselho Nacional de Segurança Alimentar; 2007.

4. Rose D. Economic determinants and dietary consequences of food insecurity in the United States. J Nutr 1999; 129:517S-20S

5. Santos SMC, Santos LMP. Avaliação de políticas públicas de segurança alimentar e combate à fome no período de 1995-2002. 1 - Abordagem metodológica. Cad Saúde Pública 2007; 23:1029-40.

6. Kaiser LL, Melgar-Quiñonez HR, Lamp CL, Johns MC, Sutherlin JM, Harwood JO. Food security and nutritional outcomes of preschool-age Mexican-American children. J Am Diet Assoc 2002; 102:924-9.

7. Kaiser LL, Melgar-Quiñonez HR, Townsend MS, Nicholson Y, Fuji ML, Martin AC, et al. Food insecurity and food supplies in Latino households with young children. J Nutr Educ Behav 2003; 35 : 148-53.

8. Nord M, Andrews M, Carlson S. Household food security in the United States 2005. Washington DC: United States Department of Agriculture, Economic Research Service; 2006. (Food Assistance and Nutrition Research Report, 29).

9. Tarasuk VS, Beaton GH. Women's dietary intakes in the context of household food insecurity. J Nutr 1999; 129:672-9.

10. Nnakwe N, Yegammia C. Prevalence of food insecurity among households with children in Coimbatore, India. Nutr Res 2002; 22:1009-16.

11. Segall-Corrêa AM, Pérez-Escamilla R, Maranha LK, Sampaio MFA, Yuyama L, Alencar F, et al. Projeto: acompanhamento e avaliação da segurança alimentar de famílias brasileiras: validação de metodologia e de instrumento de coleta de informação. Campinas: Departamento de Medicina Preventiva e Social, Universidade Estadual de Campinas/Organização Pan-Americana da Saúde/Ministério da Saúde; 2004. (Relatório Técnico).

12. Instituto Brasileiro de Geografia e Estatística. Pesquisa Nacional por Amostra de Domicílios PNAD: suplemento sobre segurança alimentar. Rio de Janeiro: Instituto Brasileiro de Geografia e Estatística; 2004.

13. Centro Brasileiro de Análise e Planejamento/Ministério da Saúde. Pesquisa Nacional de Demografia e Saúde da Criança e da Mulher: PNDS 2006: dimensões do processo reprodutivo e da saúde da criança. Brasília: Ministério da Saúde; 2009.
14. Burlandy L, Salles-Costa R. Segurança alimentar e nutricional: concepções e desenhos de investigação. In: Kac G, Sichieri R, Gigante D, organizadores. Epidemiologia nutricional. Rio de Janeiro: Editora Fiocruz; 2007. p. 485-502.

15. Matheson DM, Varady J, Varady A, Killen JD. Household food security and nutritional status of Hispanic children in the fifth grade. Am J Clin Nutr 2002; 76:210-7.

16. Pimentel PG, Sichieri R, Salles-Costa R. Insegurança alimentar, condições socioeconômicas e indicadores antropométricos em crianças da região metropolitana do Rio de Janeiro/Brasil. Rev Bras Estud Popul 2009; 26:283-94.

17. Salles-Costa R, Pereira RA, Vasconcellos MTL, Veiga GV, Marins VMR, Jardim BC, et al. Associação entre fatores socioeconômicos e insegurança alimentar: estudo de base populacional na região metropolitana do Rio de Janeiro, Brasil. Rev Nutr 2008; 21 Suppl:99s-109s.

18. Marín-León L, Segall-Corrêa AM, Panigassi G, Maranha LK, Sampaio MFA, Pérez-Escamilla R. A percepção de insegurança alimentar em famílias com idosos em Campinas, São Paulo, Brasil. Cad Saúde Pública 2005; 21:1433-40.

19. Salles-Costa R, Antunes MML, Mello MA, Sichieri R. Comparação de dois programas computacionais utilizados na estimativa do consumo alimentar de crianças. Rev Bras Epidemiol 2007; 10:267-75.

20. Secretaria de Políticas de Saúde, Ministério da Saúde/Organização Pan-Americana da Saúde. Guia alimentar para crianças menores de dois anos. Brasília: Ministério da Saúde/Organização Pan-Americana da Saúde; 2002. (Série A. Normas e Manuais Técnicos).

21. Philippi ST, Cruz ATR, Colucci ACA. Pirâmide alimentar para crianças de 2 a 3 anos. Rev Nutr 2003; 16:5-19.

22. Institute of Medicine, National Research Council. Dietary reference intakes for energy, carbohydrate, fiber, fat, fatty acids, cholesterol, protein, and amino acids (macronutrients). Washington DC: National Academies Press; 2002.

23. Coordenação-Geral da Política de Alimentação e Nutrição, Secretaria de Atenção à Saúde, Ministério da Saúde. Guia alimentar para a população brasileira. Promovendo a alimentação saudável. Brasília: Ministério da Saúde; 2006. (Série A. Normas e Manuais Técnicos).

24. Fávaro T, Ribas DLB, Zorzatto, JR, Segall-Corrêa AM, Panigassi G. Segurança alimentar em famílias indígenas Teréna, Mato Grosso do Sul, Brasil. Cad Saúde Pública 2007; 23:785-93.

25. Segall-Corrêa AM, Salles-Costa R. Novas possibilidades de alimentação a caminho? Democracia Viva 2008; 39:68-73.

26. Panigassi G, Segall-Corrêa AM, Marin-León L, Pérez-Escamilla R, Maranha LK, Sampaio MFA. Insegurança intrafamiliar e perfil de consumo de alimentos. Rev Nutr 2008; 21 Suppl:135s-44s. 
27. Batista Filho M, Rissin A. A transição nutricional no Brasil: tendências regionais e temporais. Cad Saúde Pública 2003; 19 Suppl 1:S181-91.

28. Skalicky A, Meyers AF, Adams WG, Yang Z, Cook JT, Frank DA. Child food insecurity and iron-deficiency anemia in low-income infants and toddlers in the United States. Matern Child Health J 2006; 10:177-85.

29. Borges CVD, Veiga APB, Barroso GS, Jesus EFO, Serpa RFB, Moreira S, et al. Associação entre concentrações séricas de minerais, índices antropométricos e ocorrência de diarréia entre crianças de baixa renda da região metropolitana do Rio de Janeiro. Rev Nutr 2007; 20:159-69.

30. Levy-Costa RB, Monteiro CA. Consumo de leite de vaca e anemia na infância no Município de São Paulo. Rev Saúde Pública 2004; 38:797-803.

31. Instituto Brasileiro de Geografia e Estatística. Pesquisa de Orçamentos Familiares 2002-2003: análise da disponibilidade domiciliar de alimentos e do estado nutricional no Brasil. Rio de Janeiro: Instituto Brasileiro de Geografia e Estatística; 2004.

32. Tuma RCFB, Costa THM, Schmitz BAS. Avaliação antropométrica e dietética de pré-escolares em três creches de Brasília, Distrito Federal. Rev Bras Saúde Matern Infant 2005; 5:419-28.

33. Aquino RC, Philippi ST. Consumo infantil de alimentos industrializados e renda familiar na cidade de São Paulo. Rev Saúde Pública 2002; 36:655-60.
34. Barbosa RMS, Croccia C, Carvallho CGN, Franco VC, Salles-Costa R, Soares EA. Consumo alimentar de crianças com base na pirâmide alimentar brasileira infantil. Rev Nutr 2005; 18:633-41.

35. Oliveira LPM, Assis AMO, Pinheiro SMC, Prado MS, Barreto ML. Alimentação complementar nos primeiros dois anos de vida. Rev Nutr 2005; 18:459-69.

36. Feinberg E, Kavanagh PL, Young RL, Prudent N. Food insecurity and compensatory feeding practices among urban black families. Pediatrics 2008; 122:e854-60.

37. Monteiro CA, Conde WL. Tendência secular da desnutrição e da obesidade na infância na cidade de São Paulo (1974-1996). Rev Saúde Pública 2000; 34(6 Suppl):52-61.

38. Santos JV, Gigante DP, Domingues MR. Prevalência de segurança alimentar em Pelotas, Rio Grande do Sul, Brasil, e estado nutricional de indivíduos que vivem nessa condição. Cad Saúde Pública 2010; 26:41-9.

39. Salles-Costa R, Barroso GS, Mello MA, Antunes MML, Yokoo EM. Sources of variation in energy and nutrient intakes among children from six to thirty months old in a population-based study. Cad Saúde Pública 2010; 26:1175-86.

Recebido em 08/Out/2009

Versão final reapresentada em $02 / \mathrm{Jul} / 2010$

Aprovado em 09/Jul/2010 